\title{
CULTURAS, ETNICIDADES Y AFLICCIONES. PERSPECTIVAS AMERINDIAS EN LA SALUD INTERCULTURAL
}

\author{
Gerardo Fernández Juárez \\ Facultad de Humanidades de Toledo \\ Universidad de Castilla - La Mancha \\ Gerardo.FJuarez@uclm.es
}

ORCID iD:https://orcid.org/0000-0001-5129-4473

Recibido: 20 de marzo 2018; Aceptado 22 enero 2019

Cómo citar este artículo/Citation: Fernández Juárez, Gerardo (2019), “Culturas, etnicidades y aflicciones. Perspectivas Amerindias en la Salud Intercultural", Asclepio, 71(1): p259. https://doi.org/10.3989/asclepio.2019.11

RESUMEN: El artículo analiza la incorporación y el reconocimiento de la variable étnica y cultural en la perspectiva conceptual sobre el abordaje de la salud en pueblos amerindios, circunstancia promovida desde instituciones como la OMS y su sede americana OPS. El concepto de persona o el uso diverso de la pertenencia étnica deben ser considerados con suficiente flexibilidad y perspicacia crítica para no caer en criterios monolíticos esencializadores que desvirtúen las posibilidades y los beneficios que dicha perspectiva intercultural puede propiciar en el acceso a la salud de los pueblos amerindios.

PALABRAS CLAVE: Salud intercultural; etnicidad; pueblos amerindios.

\section{CULTURES, ETHNICITIES AND AFFLICTIONS. AMERINDIAN PERSPECTIVES ON INTERCULTURAL HEALTH}

ABSTRACT: This article analyzes the incorporation and acknowledgement of the ethnic and cultural variable in the conceptual perspective on Amerindian people's health, to be considered by institutions such as the World's Health Organization (WHO) and its American headquarters or Pan American Health Organization (PAHO). Questions such as the concept of person, prevalent ailments and the various uses of ethnical belonging must be considered with flexibility and critical insight in order to avoid monolithic, pigeonholing criteria which detract from the possibilities and benefits that such intercultural perspective can bring to Amerindian people's access to health.

KEY WORDS: intercultural health; ethnicity; Amerindian people. 
Hace más de medio siglo que Gonzalo Aguirre Beltrán (1955) publicara su conocida monografía "Programas de Salud en la Situación Intercultural". Desde aquel entonces los programas de postgrado en salud intercultural desarrollados en América Latina han propiciado una masa crítica entre los profesionales de la salud que no siempre han priorizado las necesidades prácticas de las poblaciones que más precisan de sus atenciones, por las falencias claras en las inequidades que sufren en materia de salud, caso de los grupos amerindios y afrodescendientes. La aprobación por parte de la Organización Panamericana de la Salud (OPS/OMS) en Septiembre de 2017 de la inclusión de la variable cultural y étnica en los proyectos de salud de Naciones Unidas ${ }^{1}$ supone un verdadero hito en el reconocimiento de las modalidades locales de definición de aflicciones y terapias.

\section{“CUANDO No esté El MÉdico VEngo”}

Las diferentes iniciativas que en materia de salud intercultural se llevan realizando en América Latina, a lo largo de los años setenta, ochenta y muy especialmente desde los 90 del pasado siglo e inicios de los 2000, con resultados, al menos, discutibles o no tan exitosos como los esperados, obliga a que cualquier nueva iniciativa que se considere, debe ser analizada con la prudencia necesaria. En este sentido el respaldo institucional que la OPS /OMS ha propiciado para el reconocimiento de la variable étnica y cultural en los proyectos de salud, supone una nueva oportunidad para avanzar en la reflexión de las posibilidades que la salud intercultural puede propiciar en la aplicación de dichos proyectos desde la perspectiva de quienes deben ser sus beneficiarios: los pueblos amerindios y afrodescendientes.

Definir una conceptualización de la salud intercultural desde la perspectiva de las poblaciones indígenas no es tarea fácil y debemos huir de cualquier tentativa simplista en términos de definición reduccionista. Cuando tenemos en cuenta la incidencia que los hábitos culturales y su ambiente pueden generar en las formas de considerar la salud y la enfermedad en términos indígenas, tenemos que tener en cuenta una diversidad de perfiles y modalidades. Dentro de un mismo grupo étnico indígena, podemos encontrar diferentes sensibilidades y criterios formales a la hora de considerar la salud y la enfermedad por parte de sus miembros. Dichas diferencias pueden deberse a distintas variables que afecten a los implicados tales como la edad, el género, el ámbito rural o urbano de su socialización, el acceso y la familiaridad o no con los servicios médicos convencionales, su implicación con los modelos tradicionales de salud o no, o los planteamientos híbridos (medicina convencional y etnomedicina) en sus prácticas de "autoatención" médica. Seguramente no coincidirán en sus planteamientos de salud y sus conceptualizaciones médicas tanto un médico originario (habitualmente denominado, injustamente porque no se reconocen en el término, "curandero") como una joven madre de familia, o un chófer de colectivo, por mucho que pertenezcan a la misma comunidad. $Y$ todos ellos son igualmente indígenas. Si dentro de un mismo grupo indígena podemos encontrar diferentes formas y matices distintos sobre la concepción de la salud y de la enfermedad, la cuestión se complica más si abrimos el análisis a la diversidad de grupos amerindios. No podemos plantear ninguna aproximación a una formulación correcta en criterios interculturales en salud, si sólo valoramos la perspectiva indígena y no tenemos en cuenta a los otros actores que intervienen en el proceso. No podemos analizar en exclusividad la perspectiva de los "especialistas rituales" o los médicos originarios, debemos igualmente valorar todos los ámbitos y protagonistas vinculados con el ejercicio de la salud en contextos indígenas, entre ellos, los propios médicos y profesionales de la salud.

El concepto de cultura, cuyo proceloso análisis prosigue en las Ciencias Sociales y muy especialmente en el dominio de la Antropología Social y Cultural, es dinámico, no esencializador, no es homogéneo ni monolítico y adolece de cambios significativos a lo largo del tiempo. Los grupos culturales vivos, y por supuesto los pueblos amerindios, están sometidos a esos cambios significativos que en muchos ámbitos emanan de los procesos de globalización que los afecta. No podemos cosificar las expresiones culturales como si fueran objeto de inerme curiosidad museística. Por su parte, la biomedicina tampoco ofrece una interpretación rígida ni homogénea entre los pueblos amerindios siendo una realidad el grado de penetración que ha conseguido efectuar en los últimos decenios. De hecho forma parte de las respuestas incorporadas en los modelos de auto atención de los pueblos indígenas, de tal forma que incluso se solicita el envío de determinadas marcas de fármacos cuando se someten al proceso migratorio ( Meñaca 2006).

Dada la disparidad heterogénea de formas de vida, contextos, sistemas de producción, espacios de adap- 
tación, creencias y configuración de redes de parentesco que caracterizan la vida de los pueblos indígenas americanos en la actualidad (Chase Smith 2000) ¿podemos establecer algún criterio compartido en relación a la salud intercultural y su perspectiva desde los pueblos indígenas? Si esto es así, debemos preguntarnos....ंpodemos intentar algún formato exitoso en nuestro deseo de extraer alguna conclusión válida, alguna aplicación de la incidencia que algunas de estas manifestaciones culturales en la construcción social de la salud y de la enfermedad y en el proceso de salud/enfermedad/atención-prevención definido por Menéndez (2016), presentan para la concepción indígena de la salud desde una perspectiva intercultural? Si ubicamos en el centro de nuestros intereses al ciudadano [indígena o no indígena], como prioridad de nuestras reflexiones interculturales, creo que merece la pena intentarlo.

"Año pasado... el doctor era otro también...todos diferentes sus caracteres tienen ¿no ve? No son iguales. $Y$ los pacientes que tenían confianza me preguntaban:¿Está el doctor? Sí, le digo. - ¡Ah!, entonces volveré más tarde. Así, -cuando no está vuelvo, dicen, sí. Ahí yo sufría mucho, en esa parte. -Ahora... ¿qué hago, pues? ¿Qué voy a decir? (Cleo Alaru. Auxiliar Sanitaria aymara).

"Cuando no esté el médico vengo" De esta forma espetó una mujer aymara que acudió a la posta sanitaria de Qurpa (Provincia Ingavi del Departamento de La Paz, Bolivia) atendida por el entonces Equipo de Salud Altiplano en las pampas de Jesús de Machaqa, ante las recomendaciones por parte de las auxiliares de que el médico estaba disponible y que podía atenderla sin dificultad. "Cuando no esté el médico vengo" aquella rotunda respuesta provocaba incredulidad, asombro y profunda inquietud en el grupo de auxiliares sanitarios que desarrollaban su labor en la posta, en especial en Cleo Alaru, enfermera de dilatada presencia en la zona. La incredulidad no ocultaba cierto enfado relativo por parte de ellas, acostumbradas a bregar con situaciones similares de desconfianza por parte de los lugareños aymaras con respecto al papel y los procedimientos terapéuticos desarrollados en la pequeña unidad de salud. "Cuando no esté el médico vengo", aquella certeza que no hubo posibilidad de reconducir, aquella contundencia en la descalificación simbólica y efectiva de la medicina convencional bajo la figura del médico, supuso en aquel caso una ruptura irresoluble. La mujer buscaba en las auxiliares aymaras de la posta, familiarizadas con los conceptos indígenas sobre salud y enfermedad, la verdadera respuesta a sus aflicciones quedando el médico convencional ilegitimado para su tratamiento, de hecho el propio médico quedó al margen del conflicto, excluido, desconocedor del drama intercultural que en materia de salud se vivía a diario no sólo en la posta sino en todo el área de competencia del equipo. Según supe luego, el problema radicaba en la forma arrogante y prepotente en que dicho médico ejercía su labor en la posta, con una fuerte predisposición racista y ofensiva en su manera de tratar a los enfermos aymaras. Obviamente, no todos los profesionales de la salud actúan de esa forma inadecuada en la relación con los enfermos indígenas, ni todos los perfiles profesionales implicados en la posta eran contemplados con la misma visión negativa por parte de los comunarios. De hecho, la mujer aymara, auxiliar y enfermera, al ser igualmente sobrina de uno de los yatiris ${ }^{2}$ de la comunidad encarnaba, en sí misma, el paradigma de una salud intercultural realista y eficaz.

Los ejemplos, en la ya dilatada etnografía sobre pueblos amerindios, del rechazo sistemático a ciertos procedimientos, técnicas e infraestructuras de los servicios médicos convencionales, la crítica o el menosprecio a la llamada biomedicina o medicina científica que se refleja en los bajos rendimientos y escaso acceso a las unidades hospitalarias desplegadas en contextos indígenas, allí donde existen, nos obliga a plantearnos las razones de estos comportamientos y actitudes que están en el fondo de una sentida incomprensión sobre los modelos amerindios de concebir la salud y la enfermedad y los planteamientos interculturales que resulten más propios y adecuados.

$¿ C$ Cuantas veces expresan las comunidades amerindias, independientemente del grupo étnico al que pertenezcan, que los hospitales y centros de salud de nuestras estructuras sanitarias, (allí donde existen), no son los espacios apropiados para el cuidado de sus problemas sanitarios o que el médico simplemente carece de competencia para curarles, porque "no entiende" sus verdaderos padecimientos?.

Suele haber un cierto reconocimiento de las competencias del médico y de la medicina científica en lo que tiene que ver con el cuerpo humano, pero no en el cuidado de buena parte de las dolencias y aflicciones indígenas que precisan de otro tipo de diagnóstico y que son consecuencia directa de las concepciones indígenas sobre la salud y la enfermedad; uno y otro son por tanto verdaderos campos de significación que no podemos reducir a un único concepto o a una única versión. 
Hace unos años me comentaba desconsolado un yatiri, especialista ritual aymara, originario de la cuenca del Lago Titicaca sus valoraciones sobre la enfermedad y las actuaciones de los médicos de las unidades hospitalarias, (he respetado escrupulosamente en la cita, el uso que hace del castellano):

Cuando yo curo...ya sana pues. Una noche no más yo curo. No, no, yo no curo nada, ni dos noches ni tres noches, inada! Cuando yo curar ya comienzo a las ocho de la noche ${ }^{3}$ y hasta doce, hasta la una. Después, los enfermos, los que están mal , las gentes, cuando yo curo, tienen que dormir, una horita, dos horitas y cuando levantan, ya yo pregunto: “¿Cómo estás señora? ¿Estás sana? ¿Estás bien? ¿Estás malsita? Si... ¿estás mal? iya!, "estoy sana" ${ }^{4}$. Yo leo ${ }^{5}$ iya!, sanó. Los médicos no hacen nada. No, médicos, inada! Seguramente los médicos ya no curan, pero claro que sí , algunos con su "corpos" ( cuerpos) claro que seguramente malogrados, ese cura ya, los malogrados, ese no más curan los médicos ${ }^{6}$. Sólo que de saxras, saxras se llaman los "maliños" (malignos) saxra, ñanqha ${ }^{7}$, pero ...usted sabe ¿no ve? Esas clases, saxra, diablo iclaro!, algunos dicen Satanás ; de saxra siempre agarran pues sus ajayus, sus animus, su espiritu , siempre ${ }^{8}$.Eso no más, pero cuando los saxras entran los cuerpos, aquí, el corazón, el corazón entra ya, todas , todas partes le entra ${ }^{9}$. Cuando algunos yatiris, cuando también...jigual! , pero no saben captar nada. Para sí están curando (en vano), están frotando todas partes ${ }^{10}$....iya! No, no sana, no sana, así es.

La información de Manuel Chura es reveladora; manifiesta entre otras cosas una disociación importante, desde la perspectiva aymara, entre las capacidades del médico convencional y las suyas que como "médico originario" sabe discernir lo que son las "verdaderas enfermedades" , las consideradas letales y que mayores aflicciones producen en la gente que trata, frente a las preocupaciones del médico. Como dice, el médico tiene competencias en curar el "cuerpo", casi lo expresa con cierta conmiseración justificando la escasa importancia que le otorga, frente al conocimiento de las "otras enfermedades" de las que el médico no sabe. La descripción que hace sobre la patología del "susto" en términos aymaras, en la que entran en juego como causantes las entidades tutelares del Altiplano y su localización anatómica en el corazón ${ }^{11}$, resulta conocido en la etnografía local. Obviamente se trata de la conceptualización de un "experto" habituado a vérselas con los conceptos aymaras de salud y enfermedad en su versión más precisa de la Cuenca del Lago Titicaca. No todos los pobladores aymaras de la zona conocen o podrían expresar de igual forma dichas contradicciones entre los modelos médi- cos aymaras y los de la medicina convencional; precisamente su mayor familiaridad con los problemas médicos y las lógicas terapéuticas validan la utilidad y calidad de su testimonio.

¿Cómo acercarnos entonces a un modelo conceptual que clarifique el "concepto" o "conceptos" de salud de los pueblos amerindios desde una perspectiva intercultural?.

Para ello, como nos ha adelantado el yatiri Manuel Chura en su expresivo relato, donde vincula órganos anatómicos con entidades anímicas y seres excepcionales del Altiplano, debemos acercarnos en primer lugar a la evaluación del concepto de "persona" o "ser humano" sin cuyo conocimiento pormenorizado, cualquier aproximación a los ámbitos de la salud y de la enfermedad en los pueblos amerindios resulta imposible.

Ya lo explicó hace tiempo James Clifford, uno de los representantes de la llamada Antropologia Postmoderna:

Decir que el individuo está culturalmente constituido se ha convertido en un axioma. Estamos acostumbrados a oir que la persona en Bali o entre los hopi o en una sociedad medieval es diferente de los individuos de la Europa burguesa o de la Norteamérica moderna (esto es que tiene diferentes experiencias de tiempo, espacio, parentesco, identidad corporal). Presuponemos, casi sin discusión, que un sujeto pertenece a un mundo cultural específico tanto como que habla un lenguaje nativo: un sujeto, una cultura, un lenguaje. [...] la idea de que la individualidad se articula dentro de mundos de significación que son colectivos y limitados no está en tela de juicio (Clifford 1995, p.119).

Si deseamos aproximarnos a una concepción intercultural en salud por parte de los pueblos amerindios es incuestionable, por su utilidad médica y metodológica, analizar el concepto de persona, no sólo por sus alusiones a las representaciones corporales sino al ámbito de la naturaleza de las propias dolencias y aflicciones que en clave cultual se vinculan estrechamente con la salud y la enfermedad de los individuos.

\section{EL CONCEPTO DE "PERSONA" O "SER HUMANO" EN LAS POBLACIONES AMERINDIAS}

Los sistemas o modelos de representación amerindios del cuerpo y de la persona constituyen un ejemplo más de la imposibilidad de considerarlos meros avatares nuestros, como si los pueblos ameri- 
canos se vieran igualmente constreñidos en las maneras de su comprensión y representación por nuestros propios sentidos ${ }^{12}$. Es precisamente en esos modelos de representación donde tienen cabida las soluciones amerindias sobre la aflicción y la enfermedad de sus gentes:

Cuerpos descuartizados, cenizas de una hoguera encendida con sus pedazos, avatares celestes de sus órganos, huesos molidos y amasados, pechos convertidos en perros, orificios obstruidos, sangre que tiñe el arcoíris, penes extensos, vulvas voraces, vísceras flotantes en el agua de los ríos, son imágenes que impregnan los mitos amerindios y forman una espiral de muerte y vida que nos succiona y traslada a los tiempos fundacionales del mundo, cuando el universo se formó de fragmentos del cuerpo humano y cada una de las partes de éste remitía a una montaña o a una constelación, o a un árbol o a un pájaro. En aquellos tiempos, que hoy son los de cada día para el chamán todo era cuerpo y el cuerpo no era nada, o casi nada. Los mitos muestran la continuidad entre las partes del cuerpo y los seres naturales. Entre las dos series, la anatómica y la cósmica, hay sugestivas analogías formales e imprevisibles continuidades narrativas. Por ejemplo la semejanza postulada por los chorote entre los intestinos del gigante Kiswet y cierta clase de lianas o en la conversión de dos pechos femeninos en un par de perros. Es la exploración sistemática de los puntos de contacto o fricción entre la serie formada por las partes y los órganos del cuerpo y la totalidad de los seres del mundo lo que permite al chamán curar las aflicciones de las almas y los cuerpos indios (Gutiérrez Estévez 2010, p.16).

Los relatos míticos, las prácticas ceremoniales, las estrategias rituales, los códigos simbólicos asociados a las terapias, son todos ellos criterios de representación y proyecciones corporales muy frecuentes en las tradiciones amerindias. La sorpresa que nos produce en nuestras categorías cartesianas resulta completa dificultando la comprensión de los modelos indígenas que interactúan en la configuración de un concepto razonable sobre salud en criterios interculturales. Quizá nuestra sorpresa se acentúe todavía más si tenemos en cuenta el criterio de los mayas tzeltales de las serranías centrales de Chiapas quienes consideran el cuerpo de una manera singular como nos muestran los destacados informes etnográficos de Pedro Pitarch (1995; 2010):

Los indígenas tzeltales-como los mayas de manera más general-tienen no un cuerpo, sino dos. Llegué a esta conclusión de manera relativamente fortuita. Mientras trabajaba con cantos chamánicos de curación. En estos se emplean dos términos -bak'etal y winkilel- los cuales traduje durante bastante tiempo por "cuerpo", hasta que caí en la cuenta de que tienen significados diferentes. Después las conversaciones con mis informantes indígenas me confirmaron y aclararon esas diferencias. En general, la radical distinción entre lo que podríamos llamar un cuerpo carnal-un objeto físico inerte- y un cuerpo fenoménico- un medio para relacionarse con otros seres humanos- nos exige repensar las ideas indígenas sobre la corporalidad. Pero también nos obliga a recomponer un poco el concepto indígena de persona (Pitarch 2010, p. 176).

Si ya no se habla de "un cuerpo", sino de "dos" cómo en el caso tzeltal....¿¿dónde proyectar las habilidades y competencias de la biomedicina académica? ¿dónde cristalizar y cómo explicar los conocimientos terapéuticos adquiridos sobre una anatomía científica que parece no encajar con el pensamiento médico indígena? Probablemente la incidencia corporal de la medicina científica supone un rasgo no compartido por diferentes expresiones médicas amerindias, lo que constituye sin duda un serio reto a nuestro marco ideológico terapéutico.

La vinculación de las representaciones corporales amerindias con expresiones de naturaleza mítica, ceremonial de corte simbólico es una constante, al tiempo que las alusiones a la naturaleza endógena o exógena de las enfermedades y sus causas.

En el caso aymara el término jaqi equivale a persona, "ser humano" en su completa expresión. Se aplica a los que han demostrado madurez personal y responsabilidad comunitaria y es considerado como un camino, taqui. Las personas somos, desde la perspectiva aymara, el resultado de un equilibrio configurado por un cuerpo físico y un conjunto de entidades anímicas que poseen diferentes características cuyo correcto acomodo implica el bienestar de la persona. Cuando ese equilibrio es roto, generalmente por el extravío de alguna de esas entidades anímicas, surge la enfermedad o la aflicción en diferentes grados de importancia que exigen la actuación de un médico originario para restituir la salud del enfermo. La expresión de estas enfermedades prevalentes en zonas amerindias y directamente ligadas a los modelos del llamado proceso de "salud/enfermedad/atención", son sentidas en toda su rotundidad como tales por parte de la gente, aunque a veces la literatura médica y antropológica las camufle y en cierto sentido menosprecie, en la expresión "síndromes de filiación cultural”13.

Si queremos acercarnos al conocimiento de un modelo conceptual sobre la salud en términos amerin- 
dios debemos tener muy en cuenta cual es la concepción de persona en el área cultural que se trate y cómo afecta a su consideración estas enfermedades cuyas lógicas no responden al modelo biomédico y de cuyas expresiones terapéuticas y cuidados podemos obtener conclusiones y valoraciones muy útiles en términos de la propia medicina convencional.

En la cuenca del Lago Titicaca tres son las entidades anímicas que poseen los jaqi aymaras, según relatan los pobladores; de adentro hacia fuera y de mayor a menor importancia se denominan respectivamente ajayu, animu y kuraji. Los seres humanos del Altiplano aymara, están constituidos por tanto, por un cuerpo carnal y sus tres "sombras". Son denominadas kimsa ch'iwi, las tres "sombras" ${ }^{14}$ gemelas, (entre sí y con respecto a su propietario, por cuanto constituyen su "doble" animado ${ }^{15}$ ), que de hecho se ven afectadas con su pérdida provocando aflicciones y enfermedades a la persona que lo sufre.

La solución terapéutica suele acompañarse por la intervención del yatiri (especialista ritual) en la recuperación de estas "sombras" que se extravían y que hay que reintegrar, reincorporar a la unidad corporal y anímica del enfermo, por cuanto lo que sucede a estas entidades anímicas extraviadas influye directamente en la mecánica del cuerpo de su dueño, alterando sus funciones y ocasionando diferentes enfermedades.

El proceso terapéutico de restitución de la salud en estos casos está lejos del modelo médico occidental y sus estrategias. Son procedimientos de tipo simbólico y ceremonial que responden a las lógicas propias de los conceptos amerindios, en este caso aymaras, sobre la salud con expresiones fundadas de la llamada eficacia simbólica (Lévi Strauss 1987 [1949], p. 211-227).

Lo que hay que tener presente es que no se trata de "remedos folklóricos" si no de verdaderas estrategias terapéuticas y que así son entendidas no sólo por el médico indígena originario, sino por el propio enfermo y sus familiares. No podemos caer en el prejuicio etnocéntrico de descalificación de estos procedimientos, simplemente porque no los comprendemos o porque colisionan con la lógica del proceso de inferencia característico del modelo biomédico. Si queremos definir y comprender, o al menos acercarnos, a un modelo conceptual amerindio sobre la salud y la enfermedad en clave intercultural, debemos habituarnos a este tipo de intervenciones y propuestas terapéuticas donde funcionan criterios de eficacia simbólica, en las diferentes áreas culturales de América Latina.
El modelo es similar, en el fondo, al existente en otras realidades culturales amerindias con especial énfasis en los pueblos indígenas de Mesoamérica ( $\mathrm{Pi}$ tarch 1995; Martínez González 2007, 2011) y que no sólo son compatibles con la dolencia conocida como "susto"16. De hecho, buena parte de las actuaciones terapéuticas de los médicos originarios, en el caso aymara, implican procedimientos de reforzamiento de estas entidades anímicas para que estén sólidamente vinculadas al doliente, de cuyo armazón conjunto, depende el bienestar de la persona.

Lógicamente, todo lo que afecte a la estabilidad y dinámicas de relación de estas entidades anímicas supondrá diferentes grados de aflicción en la persona, que pueden redundar en patologías graves, (en términos amerindios), según las entidades afectadas, las causas de origen de la dolencia y el tiempo de demora en su restitución. Nos sorprenderá quizá conocer que las unidades hospitalarias convencionales son causa frecuente de "susto" entre quienes acuden a las consultas, precisamente por las características habituales de actuación y los procedimientos terapéuticos que tienen lugar en dichos centros, como veremos más adelante.

Una de las enseñanzas a tener en cuenta es que el concepto de persona o ser humano en las poblaciones amerindias guarda una relación de estilo con respecto a las patologías y los conceptos de salud y enfermedad definidos por el propio grupo étnico, con sus caracteres específicos (Gutiérrez Estévez 2003, p. 9; Ackerknecht 1986, p. 27).

\section{SALUD INTERCULTURAL EN POBLACIONES AMERINDIAS. ALGUNAS CONCLUSIONES}

Estar sano o enfermo en las sociedades humanas, no depende sólo de la estructura celular y de los componentes orgánicos del cuerpo sino que cada cultura decide dar sentido a lo que entiende por salud y enfermedad así como a las estrategias curativas correspondientes. En las poblaciones amerindias existe un complejo conjunto de formulaciones culturales sobre enfermedades, los llamados "síndromes de filiación culturales" que hemos visto, que recogen aspectos diferenciados de su realidad cultural. Así las cosas, determinadas patologías o aflicciones son resueltas mediante la consideración que se hace del ser humano y sus expectativas funcionales. No tiene sentido la parcelación orgánica, mirar "adentro" del cuer- 
po o preocuparse por un órgano específico. Los rituales terapéuticos empleados por diferentes grupos étnicos americanos hacen alusión a modelos simbólicos de percepción y concepción del cuerpo y del ser humano en situación de eficacia social, junto con su estructura social y de parentesco, así como las diferentes cosmovisiones que, según cada caso, se ven afectadas. Es por eso que buena parte de los procedimientos terapéuticos son de carácter ritual y simbólico de forma paralela al conocimiento prolijo de especies naturales que emplean en su abundante herbolaria y farmacopea.

Cualquier propuesta reduccionista de definición sobre un concepto indígena de salud con perspectiva intercultural, dada la gran diversidad "intra" e "intercultural" que podemos encontrar al respecto en los diferentes contextos señalados resultaría abiertamente incompleta e insatisfactoria, pero, algún resquicio nos queda para poder plantear (siempre con prudencia y aceptando las diferentes versiones y matices), alguna característica útil no sólo para pensar en los modelos indígenas de salud, sino para tenerlo en cuenta en las aplicaciones de las políticas interculturales en salud en pueblos indígenas .

El ejemplo etnográfico de los aymaras del Altiplano boliviano que hemos analizado en el artículo nos ofrece un adecuado marco de reflexión para poner a prueba nuestra capacidad analítica en la caracterización de un modelo de salud intercultural que emane de sus peculiaridades y que a la vez pueda servirnos como referente en otros casos. Destaquemos varios aspectos significativos.

Los conceptos de persona o "ser humano" resultan claves en la definición de la salud en términos indígenas puesto que nos habitúan a los sistemas de representación corporales y de las entidades anímicas que incorporan, que son los referentes implicados en la mayor parte de las enfermedades, dolencias y aflicciones de los pueblos amerindios. Muestran su vinculación con las enfermedades prevalentes definidas culturalmente (los síndromes de filiación cultural) ofreciendo consideraciones de estilo coherentes con la significación social de la enfermedad, la etiología de las dolencias así como con los sistemas terapéuticos. Las enfermedades prevalentes en clave cultural refuerzan la consideración de estilo específica del "concepto de persona", con diferencia de detalles descriptivos y matices, pero con un acuerdo razonable sobre tipología de enfermedades, diagnósticos, etiologías y tratamientos. La salud de los pueblos amerin- dios está implicada con el entorno en el que viven y su respeto, (a veces reforzado desde una perspectiva simbólica y ceremonial), de forma que cualquier alteración del mismo implica respuestas de aflicción y dolencia, del mismo modo que la alteración de alguna de las pautas de índole moral con que se dotan en el funcionamiento de sus comunidades. La salud se trata y restituye en casa y en la comunidad. No existe, en la mayor parte de los pueblos amerindios y en su concepción de salud, un espacio específico de curación exógeno a la comunidad como es el hospital con todo el bagaje simbólico y cognitivo que supone. La importante penetración de la biomedicina de los últimos decenios entre los pueblos amerindios todavía no ha resuelto de forma significativa este débito, de tal forma que la integración de las unidades hospitalarias en la vivencia cotidiana de las comunidades indígenas, sigue siendo más una excepción que la regla. Este aspecto amplía las inequidades de los pueblos indígenas en el acceso a los servicios de salud y constituye un estigma importante en lo que supone de exclusión y pobreza. Junto a este dato, el "edificio cognitivo" del hospital, allí donde existe (tal y como hemos visto en el documento), configura distintas expresiones de inseguridad, miedo, o violencia que hace que los servicios hospitalarios estén infravalorados, en ocasiones, por los grupos amerindios, quienes a veces hacen clara ostentación de no utilizarlos o necesitarlos. La salud en términos indígenas, resultado de esa relación de equilibrio corporal, doméstico y comunitario, se desempeña sin las condiciones propias de la burocracia hospitalaria. El mejor modelo de expresión de la salud en términos indígenas es la unidad doméstica y residencial, la casa y la familia, donde se realizan las curaciones precisas del enfermo al amparo del hogar, con la implicación de todo el ámbito doméstico, con el ejercicio de los especialistas locales, médicos originarios que se desplazan a la casa del enfermo y los modelos de autoatención en los que pueden aparecer diferentes signos propios de la biomedicina ( fármacos residuales, antibióticos...etc.) junto a la herbolaria local y los procedimientos rituales; con el amparo eficaz de los bagajes culturales propios en las definiciones locales del modelo de "salud, enfermedad, atención". La salud se recupera de forma individualizada y personalizada en lo que respecta a la atención del enfermo, pero con una dinámica social en la que participa y decide toda la familia e incluso la comunidad. En la lengua originaria, sin premuras de tiempo, con dedicación exclusiva cuando se precisa de la atención de un especialista local y respetando las pautas de repre- 
sentación corporal y del ser humano, así como el papel otorgado a la familia en la toma de decisiones.

Los modelos de salud indígenas dudan de las aperturas corporales y se expresan, habitualmente, sin abrir el cuerpo, sin abrir la comunidad, sin abrir la casa, sin abrir el entorno. Todos estos modelos cognitivos son expresión simbólica de la enfermedad que se introduce en cada uno de los ámbitos que engloban el resto (cuerpo, familia, aldea, comunidad, entorno) y que adolecen un carácter externo, foráneo, extracorpóreo o extracomunitario. Habrá que ver en cada caso la presencia o no de grupos étnicos más o menos proclives a las aperturas corporales y culturales en cada caso como suele suceder entre los amazónicos.

La salud en términos indígenas es multifactorial y obliga a la consideración de terapias así mismo multifactoriales en la resolución de las crisis y aflicciones que presentan expresiones rituales signadas por la eficacia simbólica. Las lenguas originarias se constituyen, en sí mismas, en vehículos de expresión de la salud a través del relato vivencial de la dolencia: el relato narrado del acontecimiento que resulta en aflicción es esencial para la recuperación del enfermo. Lo foráneo es causa de sospecha y origen de enfermedades ya sea en las expresiones lingüísticas, hábitos culturales, alimentación...etc. Lo extraño debilita la comunidad, el entorno doméstico y el criterio integrador de la persona mediante las aflicciones que genera.

Como nos han indicado ( $\mathrm{y}$ a veces reprochado) los profesionales de la salud que trabajan en contextos indígenas es imposible contribuir desde su perspectiva a restituir la salud de los enfermos indígenas si no se cuenta con medios apropiados, es decir que la llamada "salud intercultural" no puede constituir un burladero para evitar la inversión económica en parámetros de calidad de vida de los pueblos indígenas anclados en una situación lamentable de pobreza sistémica en la mayoría de los casos. Tampoco puede servirnos de excusa para justificar una atención sanitaria de mala calidad bajo su atractivo escaparate.

Cualquier iniciativa en salud intercultural debe ser sugerida, refrendada y auspiciada por las comunidades indígenas y no sólo por intelectuales o políticos al uso, si queremos que tenga impacto en la resolución de sus conflictos de salud. En la reunión que mantuvimos con el Viceministro de Medicina Tradicional e Interculturalidad de Bolivia en 2009, el representante de salud originaria del Departamento de La Paz, líder aymara, expresaba ofuscado que ellos no sabían qué era eso de la salud intercultural, que nadie se lo había explicado a ellos y lo sentían como una amenaza por parte del Estado quien, consideraban, iba a dedicarse a certificar a los médicos originarios. Tampoco entendían las razones de los médicos $\mathrm{SAFCl}^{17}$ que decían aparecían con sus "todoterreno" por las comunidades y desaparecían sin más, de hecho aquel representante de la salud indígena exigió ante el Viceministro que se desplazaran a los comunidades "médicos de verdad", es decir de los de "mandil blanco". Me sentí consternado después de aquello...pensando en el escaso suelo social que por aquel entonces una salud intercultural indígena impulsada por decreto, "desde arriba", había conseguido en los que debieran ser sus principales beneficiarios.

La salud intercultural ha de contribuir, junto a otros parámetros sociales, económicos y políticos a mejorar los estándares de vida de los pueblos amerindios y afrodescendientes porque, si no es así, estaremos contribuyendo como cómplices insospechados de su exclusión sistemática bajo el halo políticamente atractivo que proyecta su imagen; pero para ello es imprescindible no sólo adquirir competencia intercultural, algo así como un curso acelerado en antropología médica o folclore médico, sino saber percibir la diferencia que esgrimen en concepciones, modelos, terapias y aflicciones que no podemos traducir si quiera al plano biomédico sin conocer sus propias reglas de significación. 


\section{NOTAS}

1 http://www.paho.org/hq/index.php?option=com_content\&view=article\&id=13726\%3Acountries-of-the-americas-pledge=-to-improve-the-health-of-ethnic-groups-\&catid740\%3Apress-releases\&Itemid=1926\&lang=en (Consultado el 16 de marzo de 2018)

2 Yatiri. Especialista ritual aymara, diestro en la consulta de hojas de coca, en la elaboración de ofrendas ceremoniales y en la atención de aflicciones de marcado sentido cultural (Fernández Juárez 2004a).

3 Los yatiris en su mayoría curan y realizan sus preparaciones rituales por la noche, según el horario que establece la consulta de la hoja de coca que constituye su principal sistema de diagnóstico.

4 En este relato vemos que el empoderamiento sobre la enfermedad corresponde al enfermo que es quien manifiesta al especialista si está bien o está mal y no al revés como sucede en la medicina convencional que es el médico quien nos dice si estamos ya sanos o no, hasta el punto de tener que certificarnos un "alta" médica para poder abandonar el recinto hospitalario.

5 Es preciso confirmar la etiología de la enfermedad así como la eficacia de la terapia empleada mediante una consulta postrera a la hoja de coca.

6 Los médicos muestran su eficacia en el tratamiento del "cuerpo", no así respecto a las aflicciones que habitualmente afectan a las entidades anímicas de las personas.

7 Entidades malignas de la cuenca del Lago Titicaca a las que se responsabiliza de ser causa de aflicciones, dolencias y enfermedades entre los pobladores del Altiplano lacustre.

8 Los seres humanos, los jaqi del Altiplano, poseen diferentes entidades anímicas (ajayu, animu, kuraji) susceptibles de sufrir acontecimientos o sucesos que afectan la salud de las personas. Estas entidades anímicas son apresadas y retenidas por los seres maléficos del Altiplano que las devoran paulatinamente agudizando el cuadro clínico del doliente afectado. Esa es la afectación grave que revela el yatiri Manuel Chura en su testimonio.

9 Las enfermedades provocadas por la pérdida de las entidades anímicas de los aymaras no son accesibles a la medicina académica. El corazón constituye el centro anatómico del ser humano. El valor otorgado al "centro", taypi en las diversas concepciones espaciales, religiosas y sociales aymara, le atribuyen un poder aglutinante y estabilizador (Rivière 1982), lo que ilustra otro aspecto sustancial a tener en cuenta en la conceptualización indígena sobre la salud , como es el valor social de las enfermedades y aflicciones. La pérdida de este "centro" o "corazón" supone una seria amenaza, tanto para el orden comunitario como para la salud del individuo (Bastien 1996). El centro, el equilibrio y la sabiduría son caracteres asociados a la gente "con corazón", chuymani, es decir, los ancianos.

10 Los procedimientos de higiene simbólica o "limpia" son frecuentes en la terapia aymara. Las "limpias" ceremoniales pretenden, a través de los frotamientos, eliminar la contaminación que produce la enfermedad; así se consigue alejar el "daño", la desgracia, la pena, el infortunio o la envidia que ocasionan la aflicción del doliente.

11 Es conocida en las tradiciones amerindias la importancia que se otorga, tanto en los relatos míticos como en los relatos médicos sobre las enfermedades, a ciertos órganos concretos y fluidos, "corazón", "hígado", "pulmón", "pulso", "sangre", que parecen poseer cierta autonomía, más que a una idea específicamente "corporal" (Gutiérrez Estévez 2010, p.14-15). Así sucede entre los chayahuita de lengua Cahuapana en el sector nororiental de la Amazonía peruana (González Saavedra 2010, p. 248) ; como dice Pedro Pitarch, "de hecho la principal dificultad para encontrar una correspondencia entre las vísceras indígenas y las europeas reside en que las primeras no desempeñan funciones. Los cuerpos no se enferman porque algo no funcione correctamente sino porque algo ha sido sacado o movido. Entre los muchos miedos que provoca una operación quirúrgica está la certeza de que una vez que se ha hurgado dentro del cuerpo, este ya no volverá a ser el mismo" (Pitarch 2010, p.199). Las vísceras que representan las dolencias o los usos sociales sobre la enfermedad en el caso indígena no presentan un sentido fragmentario sino que contemplan una corporalidad completa en el caso tzeltal lo que contrasta con la idea del cuerpo como "organismo" indivisible del mundo occidental (Pitarch 2010, p.200). El “cuerpo", en su sentido genérico como unidad orgánica, por el contrario, es el dominio de actuación de los conocimientos parcelados y especializados del médico occidental.

12 No es casual que la machi mapuche se cubra los ojos en plena sesión chamánica junto a los ritmos de su tambor de mano, kultrún para "ver" las causas de las enfermedades; el ch'amakani aymara busca en la oscuridad de la noche el diagnóstico más acertado que confirme lo que ha visto en las hojas de coca. Por su parte los curanderos del Norte del Perú emplean el San Pedro (Trichocereus Pachanoi) para ver "en visión" los fundamentos de las enfermedades de sus clientes; de forma similar emplean la ayahuasca (Banisteriopsis kaapi) numerosos grupos amazónicos. Recursos todos ellos que muestran la distancia significativa del uso de los sentidos en las medicinas amerindias, frente a la medicina occidental. Como indica Manuel Gutiérrez Estévez (2010, p. 9) : “El pensar en cuerpos de culturas lejanas está acompañado de continuos desafíos al sentido común, a la forma común de sentir. Los sentidos de quienes pertenecen a otras culturas parecen proveerles de un tipo de percepciones que para nosotros son desconocidas, incomprensibles o insensatas. Como si los límites de sus sentidos no estuvieran sujetos a las mismas constricciones biológicas que los nuestros y sus posibilidades perceptivas fueran diferentes. El cuerpo es el objeto primero de nuestras percepciones y las convenciones sobre su naturaleza, su uso y significación constituyen el núcleo del sentido común. Pensar en otros cuerpos no sólo nos hace perder el sentido común, sino, además, la percepción razonable de nuestro cuerpo". El recorrido del argumento no afecta sólo a los cuerpos amerindios sino igualmente a la consideración de las enfermedades que afectan a dichas representaciones corporales e igualmente a las soluciones terapéuticas planteadas por los pueblos amerindios, distantes de nuestras consideraciones al uso, como distantes 
son nuestras propias representaciones sobre el cuerpo , la persona y las aflicciones humanas.

13 Tenemos diversos ejemplos en el ámbito indígena americano de este tipo de síndromes tales como mancharisqa, jap'iqa, katxyata, (versiones quechuas y aymara sobre el "susto"), la enfermedad de la "vergüenza" entre los choles mexicanos, el "empacho" etc) (Campos Navarro (Comp.)2009; Imberton 2006; Bernand 1986; Holland 1963).

14 Así la define Berg, en su Diccionario Religioso Aymara: "Ch íwi : "sombra". La sombra es considerada como una especie de alma que le sigue al hombre" (Berg 1985, p. 55). Por su parte el jesuita Bertonio recoge en su vocabulario aymara del Siglo XVII: "Cchiuu: Sombra de la cosas" (Bertonio 1984 [1612] II, p. 88).

15 Los niños recién nacidos en el Altiplano reciben sus correspondientes $c h$ 'iwis ejemplificados en la placenta, denominada ch'iwacha, "sombra", al ser expulsada (Arnold, Yapita y Tito 1999, p. 47). En las alturas de Huancabamba en el Norte de Perú nos dice Polia (1989, p. 201): “En la persona, la "sombra" es un principio inmaterial netamente distinto del cuerpo físico: puede alejarse del cuerpo manteniendo conciencia e identidad autónoma y conservando los rasgos característicos de la persona hasta el punto que al detectar la "sombra" se puede identificar la persona a la que pertenece".

16 El caso concreto del "susto" tan extendido en toda América Latina debemos destacar la implicación que tienen

\section{BIBLIOGRAFÍA}

Ackerknecht, Erwin H. (1986), Medicina y Antropología Social, Madrid, Akal.

Aguirre Beltrán, Gonzalo (1955), Programas de salud en la situación intercultural. Obra Antropológica V. México, Fondo de Cultura Económica/Universidad Veracruzana/ Instituto Nacional Indigenista / Gobierno del Estado de Veracruz.

Arnold, Denise; Yapita, Juan de Dios; Tito, Margarita (1999), Vocabulario aymara del parto y de la vida reproductiva de las mujeres, La Paz, ILCA.

Bastien, Joseph (1996), La montaña del condor. Metáfora y ritual en un ayllu andino. La Paz, Hisbol,

Berg, Hans van den (1985), Diccionario religioso aymara, Iquitos, CETA-IDEA.

Bernand, Carmen (1986), Enfermedad, daño e ideología, Quito, Abya-yala.

Bertonio, Ludovico (1984), Vocabulario de la lengua aymara, Cochabamba. Ceres/Ifea/Musef.

Campos Navarro, Roberto (comp.) (2009), El empacho en Bolivia (1612-2009), La Paz Prohisaba/Unión Europea.

Clifford, James (1995), Dilemas de la cultura. Barcelona, Gedisa.

Fernández Juárez, Gerardo (2004a), Yatiris y ch'amakanis del Altiplano aymara. Testimonios, sueños y prácticas ceremoniales, Quito, Abya-Yala. las emociones y sus expresiones formales extremas: "las situaciones que producen las reacciones emocionales repentinas son consideradas por lo general como sustos, y muy asociadas a las dolencias por pérdida de espíritu" (Gutiérrez Estévez y Surrallés 2015, p. 14). Sobre la casuística específica del "susto" , ver entre otros (Rubel 1986; Rubel et al 1995; Fernández Juárez 2004b; Neila Boyer 2006)

17 Médicos de la especialidad de Salud Familiar Comunitaria Intercultural que constituyeron en el 2005 una de las iniciativas pioneras en refrendar de forma práctica otra forma de ejercer la medicina en comunidades indígenas, pretendidamente sin la "contaminación" simbólica de la estructura hospitalaria y sus relaciones de poder. Otra cosa parece ser los resultados obtenidos. Alguna de las críticas de los propios médicos indicaban la falta de previsión en consolidar plazas (ítems) de este perfil por parte del Ministerio, una vez realizado el período de residencia. Como vemos en el testimonio del representante aymara de los médicos tradicionales de La Paz parece que tampoco se documentó su sentido y proceder en las comunidades de actuación y así mismo entre los compañeros de profesión quienes acostumbraban a zaherir a sus compañeros formados en competencia intercultural como "médicos-yatiris", o "médicos-brujos", lo que nos habla a las claras de la inadecuada comprensión de perspectivas ajenas al monopolio de los colegios médicos convencionales en la Bolivia de entonces.

Fernández Juárez, Gerardo (2004b), “Ajayu, animu, kuraji. La enfermedad del "susto" en el Altiplano de Bolivia". En: Fernández Juárez, Gerardo (coord.), Salud e Interculturalidad en América Latina. Perspectivas antropológicas, Quito, Edit. Abya-Yala /UCLM, pp.279-304.

Gutiérrez Estévez, Manuel (2003), “El estilo de la civilización amerindia", Revista de Occidente, 269, pp. 7-24.

Gutiérrez Estévez, Manuel (2010), “Esos cuerpos, esas almas. Una introducción”. En: Gutiérrez Estévez, Manuel; Pitarch, Pedro (eds.), Retóricas del cuerpo amerindio. Madrid, Iberoamericana, Madrid, pp. 9-55.

Gutiérrez Estévez, Manuel; Surrallés, Alexandre (2015), “Afectividad, cultura y sociedad en pueblos amerindios. Una breve introducción". En: Gutiérrez Estévez, Manuel; Surrallés, Alexandre (eds.), Retórica de los sentimientos. Etnografías amerindias, Madrid, Iberoamericana, pp.9-27.

González Saavedra, Luisa (2010), “El cuerpo como referente del universo chayahuita. Se buscan culpables. Aproximación a una teoría de la persona". En: Gutiérrez Estévez; Pitarch, Ramón Pedro (eds.), Retóricas del cuerpo amerindio, Madrid, Iberoamericana, pp.227-253.

Holland, William R. (1963), Medicina maya en los Altos de Chiapas, México, Instituto Nacional Indigenista.

Imberton, Gracia (2006), “La vergüenza. Enfermedad y tensiones sociales entre los indígenas choles de Chiapas, México". 
En: Fernández Juárez, Gerardo (coord.), Salud e Interculturalidad en América Latina. Antropología de la Salud y Crítica Intercultural., Quito, Abya-Yala /UCLM, pp.257-270.

Martínez González, Roberto (2007), "Los enredos del diablo: o de cómo los nahuales se hicieron brujos", Relaciones 111, Vol XXVIII, pp. 189-216.

Martínez González, Roberto (2011), El nagualismo. México, Universidad Nacional Autónoma de México.

Menéndez, Eduardo, (2016), "Interculturalidad en salud. Dudas, limitaciones y repliegues". En: Gil García, Francisco; Vicente Martín, Patricia (coords.), Medicinas y Cuerpos en América Latina. Debates antropológicos desde la salud y la interculturalidad, Quito, Edit. Abya-Yala, pp.53-97.

Meñaca, Arantza (2006), "La "multiculturalidad" de la biomedicina. El envío de medicamentos en familias migrantes ecuatorianas". En: Fernández Juárez, Gerardo (coord.), Salud e Interculturalidad en América Latina, Quito, Edit. Abya-Yala, UCLM, AECI, JCCM, FSCICIII, pp.93-108.
Pitarch, Pedro (1995), Chùlel. Una etnografía de las almas tzeltales. México, Fondo de Cultura Económica.

Pitarch, Pedro (2010), "El problema de los dos cuerpos tzeltales". En, Gutiérrez Estévez; Pitarch, Pedro (eds.), Retóricas del cuerpo amerindio, Madrid, Iberoamericana, Madrid, pp.177-211.

Polia, Mario (1989), "Contagio" y "pérdida de la sombra". En: la teoría y práctica del curanderismo andino del Perú Septentrional: Provincias de Ayabaca y Huancabamba. Revista Anthropológica del Departamento de Ciencias Sociales, 7, Lima , Pontificia Universidad Católica del Perú, pp.195-231.

Rivière, Gilles (1982), Sabaya. Structures socio-èconomiques et représentations symboliques dans les Carangas, Bolivie. EHESS, París.

Rubel, Arthur J. (1986 [1966]), "El susto en Hispanoamérica", Arinsana, 1, pp.29-42.

Rubel, Arthur J.; O'nell, Carl; Collado, Rolando (1995 [1984]), Susto. Una enfermedad popular, México, FCE. 